\title{
Determination of Alkaline Phosphatase Level in Gingival Crevicular Fluid during Rapid Maxillary Expansion
}

\author{
Ahmad Shamim*, Waheed-ul-Hamid, Muhammad Ilyas, Samina Qadir and Ushna Ahmad \\ Department of Orthodontics, de'Montmorency College of Dentistry, Pakistan

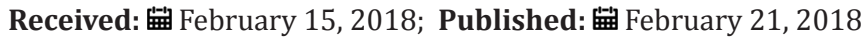 \\ *Corresponding author: Ahmad Shamim, Department of Orthodontics, de`Montmorency College of Dentistry, Pakistan
}

\begin{abstract}
Introduction: Rapid maxillary expansion (RME) is a remarkable and ideal procedure which has a long history of over 100 years. When a transverse maxillary Arch is diagnosed it is treated by doing RME. Although numerous studies have been done in last four decades on gingival crevicular fluid, no data have been reported on the repeatability of gingival crevicular fluid collection and then following its quantification procedure. The objectives of the study were to observe the changes in alkaline phosphatase (ALP) activities in gingival crevicular fluid (GCF) during rapid maxillary expansion and particularly in retention period and then explore the relationship between these changes and periodontal tissue remodeling.
\end{abstract}

Methodology: Seventeen patients with age between 9-14 years were selected according to inclusion and exclusion criteria of our study. Periodontal parameters, including probing depth, were recorded at 3 months after RME. Furthermore, the GCF ALP activity was measured at the buccal and palatal sites.

Results: Periodontal parameters were generally similar between the Testing and Control sites during the study, with the exception that probing depth underwent a slight increase at the Testing sites. It was observed that at baseline, the GCF ALP activity was similar between the buccal and Control sites; however, at 3 months of retention period a considerably increased enzymatic activity was seen at the Testing sites.

Conclusion: The results of our study concluded that in the retention phase of RME, there is reported increased GCF ALP activity observed even at the end of 3 months of retention period with no clinically considerable tissue inflammation. This increase in GCF ALP activity suggests a longer period of retention phase after maxillary expansion may be up to 6 months of duration.

Keywords: Gingival crevicular fluid; Alkaline phosphatase; Rapid palatal expansion; Bone remodeling; Hyrax expander

Abbreviations: RME: Rapid Maxillary Expansion; ALP: Alkaline Phosphatase; GCF: Gingival Crevicular Fluid; CVM: Cervical Vertebrae

\section{Introduction}

Rapid maxillary expansion (RME) is a remarkable and ideal procedure having a history of over 100 years [1]. The idea of orthopedic maxillary expansion was first described by Angell in a case report [2]. The normal Trans palatal width of maxilla ranges from $35 \mathrm{~mm}$ to $39 \mathrm{~mm}$ which suggests a bony palatal base of adequate size to accommodate a permanent dentition of normal size [3]. Expansion of the bony palatal base and width of dental arch allows us to contain extra space for the alignment of the crowded permanent dentition [4]. The expansion appliances normally produce orthopedic forces in the range of 3 to 10 pounds [5]. There

are certain etiological factors which cause Transverse maxillary constriction including genetics, environmental and functional factors. Different devices which cause maxillary expansion are Haas, Hyrax, Minnie expander, Cap Splints and recently developed acrylic bonded Rapid maxillary expander and also NiTi expanders [6].

When we do Expansion in skeletally mature patient it can produce unwanted effects like lateral tipping of posterior teeth, extrusion of teeth, periodontal membrane compression, buccal root resorption, alveolar bone bending, fenestration of the buccal cortex, 
palatal tissue necrosis, inability to open the midpalatal suture, pain, and instability of the expansion. There are certain factors that limit maxillary expansion in skeletally mature patients. These factors are related to changes with increasing age in the osseous articulations of the maxilla with the adjoining bones [7]. Gingival crevicular fluid (GCF) proves to be an exudate and the constituents present in it are derived from microbial dental plaque, inflammatory cells present in it, host tissue, and serum. Different constituents present in the GCF gives information about diseases of periodontium and they have been proved as a diagnostic indicator of any damage to the tissues but only some studies have paid attention on the aspect that the constituents of GCF can give information about remodeling of bone also [8,9]. Alkaline phosphatase (ALP) is a hydrolase enzyme and its activity has been evident in bone-forming cells, and any change in the enzyme seen in serum and bone have been used as indicator for assessing metabolism of bone in a number of diseases.

Acid and enzyme alkaline phosphatase in GCF of humans have been interconnected with the movement of teeth throughout the treatment of orthodontics and entire time period of appliance which is present in the mouth [8-12]. Elevated levels of alkaline phosphatase activities are associated with active bone formation [13]. The accurate timing of treatment in Orthodontics plays an important role in the outcome of orthopedic treatment of dental and skeletal defects in growing patients. There are unique maturational methods present which assess the skeletal maturity of the growing patients and they are based on radiographs like analysis of hand and wrist and the maturation of cervical vertebrae (CVM) method. These days other possibilities in the form of biochemical markers are obtainable and our patients can be benefitted from avoidance to unnecessary radiographic exposure. These agents are mainly involved in the growth of bone and its remodeling [14] In GCF the first enzyme which was identified is ALP and this enzyme is essential for bone mineralization. In orthodontics the activity of GCF and ALP has been suggested as a diagnostic support $[15,16]$. Therefore this study is designed to examine the changes in activities of ALP in GCF.

\section{Methodology}

This study was conducted in the department of Orthodontics de'Montmorency college of Dentistry, Lahore and the ELISA of collected samples was done at University of Health Sciences, Lahore. The sample size was 17 patients and duration of study was 01 year. Patients were selected according to the Inclusion criteria and it was Patients with narrow maxilla that requires palatal expansion. Patients having good oral hygiene and periodontal status and Patients having age between 9 to 14 yrs. The exclusion criteria were Patients with compromised periodontal status. Patients having any systemic disease like arthritis, diabetes. Patients on antibiotic or NSAIDS therapy. Patients who refuse to give consent. Patient's history, physical, systemic examination was assessed prior to study. Complete oral prophylaxis of the patient was made necessary with strict oral hygiene instructions and rinsing of mouth twice (twelve hourly) daily with 0.5 ounces of $0.2 \%$ chlorhexidine gluconate was started 1 week prior to the study and it was advised not to stop it as long the study continues.

After taking the impression study cast was made and an appliance (bonded Hyrax expander) was fabricated and cemented in to the patient maxilla. One day before cementing appliance GCF was collected from the lingual and buccal sides of lower arch using paper strips and it was taken as control group. The gingiva was massaged to activate the GCF formation before the sample collection. The patient was advised to gargle strongly with a glass of sterile water to cleanse the oral cavity. The teeth were isolated by using a self-retaining retractor, use of suction and cotton rolls. The paper points was placed lingually and buccally to obtain GCF. In case of insufficient sample, the collection of GCF sample was done frequently like twice or three times until sufficient sample obtained. After the insertion of expander the expansion was done by activating the expander and it was .25 turn in the morning and .25 turn in the evening. GCF sample was taken with same technique after insertion of appliance on day 1, day 14, and day 21 and 3 months after active phase of expansion stopped subsequently. In the control group the collection of GCF was also taken on the same days. An upper occlusal x-ray was done after 1 week to confirm expansion has started. ALP level was measured in mmol/L by ELISA technique.

\section{Statistical Analysis}

The collected data was entered and analyzed by using SPSS (Statistical Package for Social Sciences) version 20. The mean level of activity of enzyme alkaline phosphatase was calculated and the standard deviation of the mean values of the enzyme at the four sites was determined. Multivariant ANOVA analysis was applied to calculate any significance of enzyme activity among the predetermined 4 sites. A P-value $\leq 0.05$ was considered as statistical significant.

\section{Results}

Out of 17 patients, 11 (64.71\%) were males and 6 (35.29\%) were females. The mean age of patient was $12.35 \pm 1.57$ and maximum number of patients was observed at age of 12 and 14 years. The descriptive statistics of alkaline phosphatase levels are shown in Table 1. A one-way analysis of variance (ANOVA) was used to analyze the mean of alkaline phosphatase levels among five groups (Day 0, Day 1, Day 14, Day 21 and Day 03 months). There was a statistically significant difference $(\mathrm{P}<0.05)$ in mean alkaline phosphatase levels in all five groups as shown in Table 2 . The mean plot shows that mean alkaline phosphatase level increases from day 0 to day 90 (Table 3). The assumption of homogeneity of variance was violated therefore Welch and Brown-Forsythe test were used $(\mathrm{P}<0.05)$ The mean difference of mean alkaline phosphatase level between day 1 and day 0 groups is $14.824 \pm 0.95$ with a significant difference of $\mathrm{P}<0.05$. The mean alkaline phosphatase level between 
day 14 and day 0 groups is $16.29 \pm 1.56$ with a significant difference level between day 14 and day 1 groups is $1.471 \pm 1.50$ which is not of $\mathrm{P}<0.05$. The mean difference of mean alkaline phosphatase significant $(\mathrm{P}>0.05)$ (Figure 1 ).

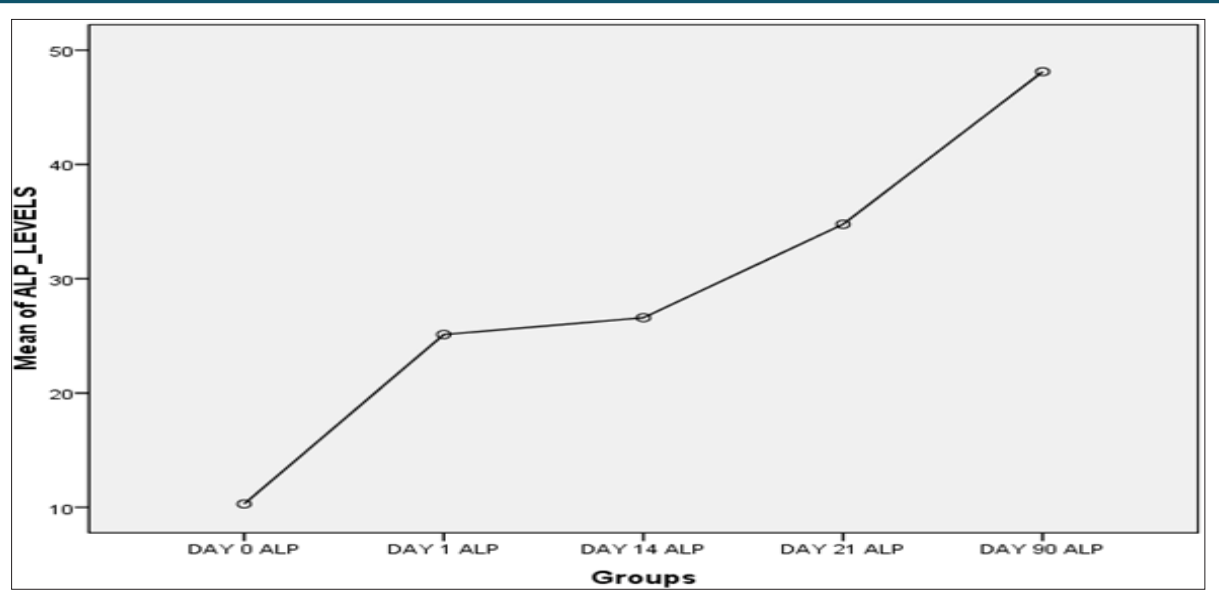

Figure 1: Mean Plot Of Alkaline Phosphatase Levels.

Table 1: Descriptive Statistics of Alkaline Phosphatase Levels.

\begin{tabular}{|c|c|c|c|c|c|c|c|c|}
\hline Alkaline Phosphatase & & & & & 95\% Confidenc & terval for Mean & & \\
\hline Levels & $\mathbf{N}$ & Mean & $\begin{array}{c}\text { Std. } \\
\text { Deviation }\end{array}$ & Std. Error & Lower Bound & Upper Bound & Minimum & Maximum \\
\hline DAY 0 ALP & 17 & 10.29 & 3.057 & .741 & 8.72 & 11.87 & 5 & 16 \\
\hline DAY 1 ALP & 17 & 25.12 & 2.497 & .606 & 23.83 & 26.40 & 20 & 29 \\
\hline DAY 14 ALP & 17 & 26.59 & 5.691 & 1.380 & 23.66 & 29.51 & 18 & 36 \\
\hline DAY 21 ALP & 17 & 34.76 & 6.078 & 1.474 & 31.64 & 37.89 & 24 & 46 \\
\hline DAY 90 ALP & 17 & 48.12 & 9.006 & 2.184 & 43.49 & 52.75 & 31 & 61 \\
\hline Total & 85 & 28.98 & 13.688 & 1.485 & 26.02 & 31.93 & 5 & 61 \\
\hline
\end{tabular}

Table 2: One-Way ANOVA

\begin{tabular}{|c|c|c|c|c|c|}
\hline ALP_LEVELS & Sum of Squares & Df & Mean Square & F & Sig. \\
\hline Between Groups & 13081.718 & 4 & 3270.429 & 98.498 & \\
\hline Within Groups & 2656.235 & 80 & 33.203 & & \\
\hline Total & 15737.953 & 84 & & \\
\hline
\end{tabular}

Table 3: Multiple Comparisons Games-Howell.

\begin{tabular}{|c|c|c|c|c|c|c|}
\hline \multirow{2}{*}{ (I) Groups } & \multirow{2}{*}{ (J) Groups } & \multirow{2}{*}{$\begin{array}{c}\text { Mean Difference } \\
\text { (I-J) }\end{array}$} & \multirow{2}{*}{ Std. Error } & \multirow{2}{*}{ Sig. } & \multicolumn{2}{|c|}{ 95\% Confidence Interval } \\
\hline & & & & & Lower Bound & Upper Bound \\
\hline \multirow{4}{*}{ DAY 0 ALP } & DAY 1 ALP & $-14.824^{*}$ & .957 & .000 & -17.60 & -12.05 \\
\hline & DAY 14 ALP & $-16.294^{*}$ & 1.567 & .000 & -20.90 & -11.69 \\
\hline & DAY 21 ALP & $-24.471^{*}$ & 1.650 & .000 & -29.34 & -19.60 \\
\hline & DAY 90 ALP & $-37.824^{*}$ & 2.307 & .000 & -44.74 & -30.91 \\
\hline \multirow{4}{*}{ DAY 1 ALP } & DAY 0 ALP & $14.824^{*}$ & .957 & .000 & 12.05 & 17.60 \\
\hline & DAY 14 ALP & -1.471 & 1.507 & .863 & -5.94 & 3.00 \\
\hline & DAY 21 ALP & $-9.647^{*}$ & 1.594 & .000 & -14.39 & -4.90 \\
\hline & DAY 90 ALP & $-23.000^{*}$ & 2.267 & .000 & -29.84 & -16.16 \\
\hline \multirow{4}{*}{ DAY 14 ALP } & DAY 0 ALP & $16.294^{*}$ & 1.567 & .000 & 11.69 & 20.90 \\
\hline & DAY 1 ALP & 1.471 & 1.507 & .863 & -3.00 & 5.94 \\
\hline & DAY 21 ALP & $-8.176^{*}$ & 2.019 & .003 & -14.01 & -2.34 \\
\hline & DAY 90 ALP & $-21.529^{*}$ & 2.584 & .000 & -29.08 & -13.98 \\
\hline
\end{tabular}




\begin{tabular}{|c|c|c|c|c|c|c|}
\hline \multirow{4}{*}{ DAY 21 ALP } & DAY 0 ALP & $24.471^{*}$ & 1.650 & .000 & 19.60 & 29.34 \\
\hline & DAY 1 ALP & $9.647^{*}$ & 1.594 & .000 & 4.90 & 14.39 \\
\hline & DAY 14 ALP & $8.176^{*}$ & 2.019 & .003 & 2.34 & 14.01 \\
\hline & DAY 90 ALP & $-13.353^{*}$ & 2.635 & .000 & -21.03 & -5.68 \\
\hline \multirow{4}{*}{ DAY 90 ALP } & DAY 0 ALP & $37.824^{*}$ & 2.307 & .000 & 30.91 & 44.74 \\
\hline & DAY 1 ALP & $23.000^{*}$ & 2.267 & .000 & 16.16 & 29.84 \\
\hline & DAY 14 ALP & $21.529 *$ & 2.584 & .000 & 13.98 & 29.08 \\
\hline & DAY 21 ALP & $13.353^{*}$ & 2.635 & .000 & 5.68 & 21.03 \\
\hline
\end{tabular}

*The mean difference is significant at the 0.05 level.

A statistically significant difference $(\mathrm{P}<0.05)$ was observed between day 21 and day 0 , day 21 and day 1, day 21 and day 14 groups with mean difference of $24.47 \pm 1.65$, 9.64 $\pm 1.59,8.17 \pm 2.01$ respectively. A statistically significant difference $(\mathrm{P}<0.05)$ was also observed between day 90 and day 0 , day 90 and day 1, day 90 and day 14, day 90 and day 21 groups with mean difference of $37.824 \pm 2.30,23 \pm 2.26,21.529 \pm 2.58,13.353 \pm 2.63$ respectively. Post Hoc concludes that among five groups (day 0, day 1, day 14, day 21 and day 90), the mean alkaline phosphatase level are statistically significant in day 1 group vs day 0 group, day 14 group vs day 0 group, day 21 group vs day 0 group, day 21 vs day 1 , day 21 vs day 14. A significant difference $(\mathrm{P}<0.05)$ was observed when day 90 was compared with day 0 , day 1 , day 14 and day 21 groups while no significant difference was seen between day 14 and day 1 groups $(\mathrm{P}>0.05)$.

\section{Discussion}

Constricted maxilla is one of the common reasons of the anomalous dentition. This anomaly may be due to different factors like genetics, environmental and functional factors $[17,18]$. It can be corrected by expansion of the palate and the most preferred method among growing patients is Rapid Maxillary Expansion [6]. The RME can be achieved with different methods which include use of haas expander, Minnie expander, Cap splints, Niti expanders. In this study we used the bonded hyrax expander [6]. Normally the RME is done in growing patients but if the subject is skeletally mature then expansion is done through surgically assisted RME [19] The normal width of the maxilla is around $35 \mathrm{~mm}$ to $39 \mathrm{~mm}$ approximately which accommodates the dentition of average size $[3,4]$. The patients which we selected for our study were evaluated and diagnosed with constricted maxilla before the commencement of the treatment. To assess the width of the maxilla we can use different methods like x-rays and cast analysis but these days bio markers are providing new options and they are considered as diagnostic markers of different anomalies like bone diseases or periodontal diseases in mouth. The advantages of biomarkers are by checking their values we may keep our patients away from extra radiographic exposures, and they signify certain agents that are concerned mainly in bone growth and remodeling [14]. Alkaline phosphatase is an enzyme which is easily detectable and it is used as a symbol of bone metabolism in numerous diseases [8,12]. As increased levels of ALP are associated with bone formation so these levels can play vital function in the onset of exact treatment timings of orthopaedic intervention. Alteration of alkaline phosphatase levels in gingival crevicular fluid is also an indicator of bone remodeling while orthodontic tooth movement $[20,12]$.

In the present study, the alkaline phosphatase levels were calculated at different times from zero hour to 21 days and then on 3 months after the active phase of expansion were over on day 21. At the experiment side, the alkaline phosphatase level reaches peak on the 14th day after placement/activation of orthodontic appliance. This finding matches with the earlier study $[21,22]$. It is detected that there is a decline in the level of alkaline phosphatase on the 21st day in the experiment side which matches with the present study. This may be due to the reply of periodontium in respond to the orthodontic force delivery over time [20]. There has been a single study available on humans which checked the action of ALP and GCF related with RME treatment and it did not mainly checked the tension sites and it mentioned a time period of only 28 days follow up, but showed the bone turnover markedly longer [21] The ALP is a enzyme which is involved mainly in bone mineralization through the process of hydrolyzing inorganic pyrophosphate, [23] and the rate of bone formation is widely associated with it [24]. Alkaline phosphatase is an activity of bone formation cells i.e. Osteoblasts.

Alteration of alkaline phosphatase levels in gingival crevicular fluid is also an indicator of bone remodeling while orthodontic tooth movement $[20,12]$. In the present study, the alkaline phosphatase levels were calculated at different times from zero hour to 21 days. At the experiment side, the alkaline phosphatase level reaches peak on the 14th day after placement/activation of orthodontic appliance. This finding matches with the previous study [21,22]. Batra and colleagues also detected a decline in the level of alkaline phosphatase on the 21st day in the experiment side which matches with the present study. This may be due to the reply of periodontium in respond to the orthodontic force delivery over time. Keeling and colleagues in a study, showed that alkaline phosphatase is also an indicator of bone remodeling while orthodontic tooth movement [25].

It is important to mention here that the results showed that GCF and ALP activity noted at the end of 3 months of retention period was still raised as we compare it with the 21 days of 
activation which showed a decline in their level. This raised level has suggested us that we should increase the retention period to more than 3 months may be up to 6 months after the removal of appliance. The results indicated that the formation of alveolar bone is still active even after the retention phase was over. This suggested that these results are comparable with other studies which have been reported earlier and even though with RME we obtain mainly the skeletal effects especially in the prepubertal phase of growth, but still we obtain dento alveolar effects too [26]. Although other studies reported that when the orthodontic treatment commences the chances of increased gingival swelling and plaque buildup is a normal phenomenon [27] so it may be a cause of increased ALP level but good hygiene of oral cavity measures during the treatment of orthodontics can decrease in plaque buildup and swelling of the gums [28]. Moreover, we can avoid major biases by doing some split mouth protocol studies that affect the GCF and ALP levels during some systemic and local metabolic conditions. We used Hyrax expanders for maxillary expansion because it does not irritate the palatal mucosa and its cleaning is very easy.

The clinical significance of the results of our study shows that even at 3 months post RME the formation of alveolar bone at the tension sites of the teeth which are supported is still alive which needs to extend retention period up to 6 months so that proper bone formation and mineralization of these sites takes place with very less chances of relapse. As after the 3 months of retention period chances of relapse are very much high even after the use of retention appliance (Transpalatal arch with extended arms) and for patients it is not feasible to wear the appliance for the second time after the relapse of expansion. Recently a study has been done by some researchers who recommended that as the bone formation and mineralization actively goes on even at 6 months so the retention period after the expansion of maxilla may be increased from 3 months to 6 months for proper formation of bone to avoid relapse [29] In our study, we checked the levels of GCF and ALP till 3 months after RME to assess formation of alveolar bone at the pressure sites of teeth which are supported. As at base line the GCF and ALP activity was alike at both testing and control sites but as the expansion started it raised significantly especially at day 14 . This activity decreased around day 21 but the results showed that GCF and ALP levels were significantly increased even at the end of 3 months of retention period and no marked tissue inflammation was seen. This enhances the support for an increase in retention period may be up to 6 months so that a very negligible chance of relapse may be noted.

\section{Conclusion}

The results of our study concluded that in the retention phase of RME, there is detection of increase in GCF ALP activity even at the end of 3 months of retention showing active bone metabolism but with no clinically significant tissue inflammation. This increase in GCF ALP activity demands a longer period of retention phase may be up to 6 months of duration before starting second phase of active treatment.

\section{References}

1. Lamparski DG, Rinchuse DJ, Close JM (2003) Comparison of skeletal and dental changes between 2 point and 4 point rapid palatal expanders. Am J Orthod Dento facial Orthopaedic 123(3): 321-328.

2. Lokesh Suria, Parul Tanejab (2008) Surgically assisted rapid palatal expansion: A literature review. Am J OrthodDentofacialOrthop 133(2): 290-302.

3. Qasim Saeed M, SajidNaeem, MueedZaigham A (2004) A Comparison of the effects of rapid maxillary expansion screw height (deep vsshallow ) on the dentoalveolar structures in maxillary constricted patients. Biomedica 20: 90-95.

4. Qasim Saeed M, Sajid Naeem, Mueed Zaigham A (2004) A Comparison of the effects of rapid maxillary expansion screw height (deep vsshallow) on the dentoalveolar structures in maxillary constricted patients. Biomedica 20: 90-95.

5. Qasim Saeed M, Haroon Qazi, Fayyaz Ahmad, Mueed Zaigham A (2009) Effects of anterior maxillary expansion. A postero-anterior cephalometric evaluation. J Pak Dent Assoc 18(3): 102-106.

6. Wendling LK, James A, McNamara JA, Franchi, Baccetti T, et al. (2004) Prospective study of the short-term treatment effects of the acrylicsplint rapid maxillary expander combined with the lower Schwarz appliance. Angle Orthod 75(1): 7-14.

7. Lokesh Suria, Parul Tanejab (2008) Surgically assisted rapid palatal expansion: A literature review. Am J Orthod DentofacialOrthop 133(2): 290-302.

8. Perinetti G, Paolantonio M, Attilio MD, Archivio DD, Tripodi D, et al. (2002) Alkaline phosphatase activity in gingival crevicular fluid during human orthodontic tooth movement. Am J Orthod Dentofacial Orthop 122(5): 548-556.

9. Lamster IB (1992) The host response in gingival crevicular fluid: potential applications in periodontitis clinical trials [Review]. J Periodontol 63(12 Suppl): 1117-1123.

10. Delmas PD (1992) Clinical use of biochemical markers of bone remodeling in osteoporosis. Bone 13: 17-21.

11. Farley JR, Hall SL, Ritchie C, Herring S, Orcutt C, et al. (1992) Quantification of skeletal alkaline phosphatase isoenzyme in canine serum. J Bone Miner Res 7(7): 779-792.

12. Insoft M, King GJ, Keeling SD (1996) The measurement of acid and alkaline phosphatase in gingival crevicular fluid during orthodontic tooth movement. Am J Orthod Dentofacial Orthop 109(3): 287-296.

13. Mishina Y, Starbuck MW, Gentile MA, Fukuda T, Kasparcova V, et al. (2004) Bone morphogenetic protein type IA receptor signaling regulates postnatal osteoblast function and bone remodeling. Journal of Biological Chemistry 279(26): 27560-27566.

14. Perinetti G, Baccetti T, Contardo L, Di Lenarda R (2011) Gingival crevicular fluid alkaline phosphatase activity as a non-invasive biomarker of skeletal maturation. Orthodontics \& craniofacial research 14(1): 44-50.

15. Rodan GA (1992) Introduction to bone biology. Bone 13(1): S3-S6.

16. Perinetti G, Paolantonio M, DAttilio M, DArchivio D, Tripodi D, et al. (2002) Alkaline phosphatase activity in gingival crevicular fluid during human orthodontic tooth movement. American Journal of Orthodontics and Dentofacial Orthopedics 122(5): 548-556. 
17. Duruk C, Sokucu O, Sezer H, Canbaye E (2004) Evaluation of nasal airway resistance during rapid maxillary expansion using acoustic rhinometry. Eur J Orthod 26(4): 397-401.

18. Ozbek M, Memikoglu T, Altug Atac A, Lowe A (2009) Stability of maxillary expansion and tongue posture. Angle Orthod 79(2): 214-219.

19. Haas AJ (1965) The treatment of maxillary deficiency by opening the midpalatal suture. The Angle orthodontist 35(3): 200-217.

20. Batra P, Kharbanda OP, Duggal R, Singh N, Parkash H, et al. (2006) Alkaline phosphatase activity in gingival crevicular fluid during canine retraction. Orthodontics \& Craniofacial Research 9(1): 44-51.

21. Wei FL, Wang CL, Liu DX, Guo J, Ke HF, et al. (2007) [Changes of aspartate aminotransferase and alkaline phosphatase activities in gingival crevicular fluid during rapid palatal expansion]. Shanghai kou qiang yi xue Shanghai journal of stomatology 16(2): 168-171.

22. DAttilio M, Filippi MR, Femminella B, Festa F, Tecco S, et al. (2005) The influence of an experimentally-induced malocclusion on vertebral alignment in rats: a controlled pilot study . Cranio 23 : $119-129$

23. Robison R, Soames KM (1924) The possible significance of hexosephosphoric esters in ossification: Part II The phosphoric esterase of ossifying cartilage. Biochemical Journal 18(3-4): 740.
24. Christenson RH (1997) Biochemical markers of bone metabolism: an overview. Clinical biochemistry 30(8): 573-593.

25. Keeling SD, King GJ, McCoy EA, Valdez M (1993) Serum and alveolar bone phosphatase changes reflect bone turnover during orthodontic tooth movement. American Journal of Orthodontics and Dentofacial Orthopedics 103(4): 320-326.

26. Lagravere MO, Major PW, Flores Mir C (2005) Long-term skeletal changes with rapid maxillary expansion: a systematic review. The Angle orthodontist 75(6): 1046-1052.

27. Trossello VK, Gianelly AA (1979) Orthodontic treatment and periodontal status. Journal of periodontology 50(12): 665-671.

28. Lundström F, Hamp SE, Nyman S (1980) Systematic plaque control in children undergoing long-term orthodontic treatment. The European Journal of Orthodontics 2(1): 27-39.

29. Perinetti G, DApuzzo F, Contardo L, Primozic J, Rupel K, et al. (2015) Gingival crevicular fluid alkaline phosphate activity during the retention phase of maxillary expansion in prepubertal subjects A split-mouth longitudinal study. Am J Orthod Dentofac 148: 90-96.

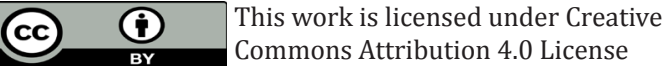

To Submit Your Article Click Here:

Submit Article
DOI: 10.32474/MADOHC.2018.01.000115

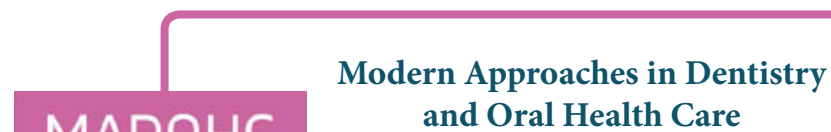

Assets of Publishing with us

- Global archiving of articles

- Immediate, unrestricted online access

- Rigorous Peer Review Process

- Authors Retain Copyrights

- Unique DOI for all articles 